\title{
The Mechanism and Quantitative Description of the Photoconductivity and Photo-EPR in Beta-rhombohedral Boron Single Crystals at $77 \mathrm{~K}$
}

\author{
D. Geist
}

Institut für Angewandte Physik der Technischen Universität Clausthal, Germany

Z. Naturforsch. 28 a, $953-964$ [1973] ; received 26 February 1973)

\begin{abstract}
The inhomogeneous excitation resulting from strong absorption of the incident light as well as the low carrier mobility resulting in nearly negligible diffusion is taken into account in converting the directly measured conductance and number of spins into a time- and intensity of illuminationdependence of the concentrations of localized states and carriers. Then a model as simple as possible is searched out which will be able to explain simultaneously the behaviour of the conductivity and density of carriers and spin carrying centres. The resulting four coupled nonlinear differential equations have been solved analytically and numerically. Within the accuracy of the measurements, the observations can be reproduced.
\end{abstract}

\section{Introduction}

Beta-rhombohedral boron is a p-type semiconductor with a rather low electrical conductivity. Under illumination sufficiently pure single crystals are photoconductive and at the same time an electron paramagnetic resonance line $(\mathrm{EPR})$ appears ${ }^{1}$. Both effects depend in the same way, but rather complicated, on the duration and intensity of the illumination and on the temperature. The present paper deals with the phenomena at $77 \mathrm{~K}$ when persistent effects dominate. Their consistent treatment is based on an appropriate energy level scheme. The properties of the levels with respect to the electron paramagnetic resonance and to conductivity, their densities, the transition probabilities between them and their occupation in thermodynamic equilibrium as well as under illumination is treated in Section 2. A comparison between the predictions derived from the theoretical model and the experiments follows in Section 3. Next, the numerical solution of the differential equations describing the time dependent occupation of the levels and the densities of the carriers is treated in Section 4. Finally, in Section 5, the physical nature of the levels involved is discussed.

It seems appropriate to state explicitly, that the EPR line appearing under illumination will probably be of an origin different from that, which is responsible for the EPR line often found with boron in the dark. This statement relies on the observation, that the "dark line" is definitely sharper $\left(1.8 \times 10^{-4}\right.$ $\mathrm{T})$ than the light induced one $\left(4 \ldots 30 \times 10^{-4} \mathrm{~T}\right.$ at $77 \mathrm{~K}$ ). This can clearly be seen from Fig. A 1, curve 2 , in the Appendix, valid for zone molten single crystals (see also ${ }^{1}$ ). In earlier measurements ${ }^{2-4}$ on polycrystals, the line in the dark was broader and could not be distinguished from the EPR-line (called A) under illumination. This line $\mathrm{A}^{2,3}$ had been attributed to "movable" carriers. This expression shall not mean, that the species responsible for the EPR must be in every case carriers (electrons resp. holes) in a conduction or valence band. These species may be carriers able to hopping from site to site thus being movable. It seems most probable, that the carriers on a site are responsible for the EPR. Earlier remarks in Refs. ${ }^{5,6}$ should be interpreted resp. corrected in this sense.

\section{The Levels}

\section{a) Traps, Donors and Acceptors}

According to Fig. 1 there are traps $\mathrm{T}$ in the density $\mathrm{T}$ characterized by the reaction

$$
\mathrm{T}^{\times}+\mathrm{e}^{-} \rightleftharpoons \mathrm{T}^{-}
$$

with $\mathrm{T}^{\times}$for a neutral and $\mathrm{T}^{-}$for a negatively charged trap and with $\mathrm{e}^{-}$for an electron.

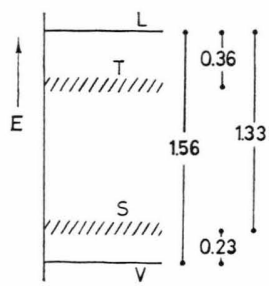

Fig. 1. Energy levels in beta-rhombohedral boron.

$\mathrm{E}$ electron energy (numbers meaning $\mathrm{eV}$ )

$\mathrm{L}$ bottom of the conduction band

$\mathrm{V}$ top of the valence band

$\mathrm{T}$ traps for electrons

$\mathrm{S}$ donors $\mathrm{D}$ and acceptors $\mathrm{A}$.

This scheme is compatible with results on the optical absorption ${ }^{10}$. 
The donors $\mathrm{D}$, density $n_{\mathrm{D}}$, with

$$
\mathrm{D}^{\times}-\mathrm{e}^{-} \rightleftharpoons \mathrm{D}^{+}
$$

and acceptors A, density $n_{\mathrm{A}}$, with

$$
\mathrm{A}^{\times}+\mathrm{e}^{-} \rightleftharpoons \mathrm{A}^{-}
$$

are assumed to have the same distance in energy $E_{\mathrm{S}}-E_{\mathrm{V}}$ from the upper edge of the valence band

$$
E_{\mathrm{S}}-E_{\mathrm{V}}=E_{\mathrm{D}}-E_{\mathrm{V}}=E_{\mathrm{A}}-E_{\mathrm{V}}=\Delta k T
$$

( $k$ Boltzmann's constant, $T$ absolute temperature).

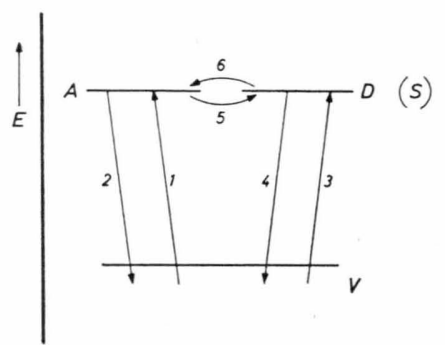

Fig. 2. Transition processes. Symbols as in Figure 1.

With respect to the magnetic behaviour (EPR), the following prescriptions are essential. Neutral donors $\mathrm{D}^{\times}$are assumed spincompensated. Singly positive charged donors $\mathrm{D}^{+}$shall be carrying a free spin. They shall be able to give EPR. As to statistics, this state is degenerate with $g_{\mathrm{fr}}=2$.

Only for definiteness singly charged acceptors $\mathrm{A}^{-}$ are assumed to be spin compensated, and neutral ones $\mathrm{A}^{\times}$degenerate (different assumptions for the acceptors would be admissible).

\section{b) Equilibrium Occupation at Low Temperatures}

The conduction band and the traps are essentially empty

$$
n \approx 0, \quad T^{-} \approx 0
$$

( $n$ electron concentration, $p$ hole concentration). Then the absence of net charge requires

$$
n_{\mathrm{A}}^{-}=p+n_{\mathrm{D}}^{+} ;
$$

assuming nondegeneracy of the holes in the valence band and introducing the appropriate Fermi occupation probabilities, the condition yields

$$
\begin{gathered}
\frac{n_{\mathrm{A}}}{1+a \exp \left\{\left(E_{\mathrm{A}}-\mu^{*}\right) / k T\right\}}=p_{0} \exp \left\{\left(E_{\mathrm{V}}-\mu^{*}\right) / k T\right\} \\
+\frac{n_{\mathrm{D}}}{1+b \exp \left\{\left(\mu^{*}-E_{\mathrm{D}}\right) / k T\right\}}
\end{gathered}
$$

or

$$
n_{\mathrm{A}}+n_{\mathrm{A}} e^{\eta}-n_{\mathrm{D}}-n_{\mathrm{D}} e^{-n}=p_{2}\left(1+e^{-\eta}\right)^{2} .
$$

It is determining the position of the Fermi level (electrochemical potential) $\mu^{\times}$.

Abbreviations are as follows

$b=1 / a=1 / 2 \quad$ according to Sect. 2 a

$p_{0}=\left(2 / h^{3}\right)\left(2 \pi m_{\mathrm{p}} k T\right)^{3 / 2} \quad\left(m_{\mathrm{p}}\right.$ effective hole mass)

$p_{2}=p_{0} b e^{-\Delta} \quad$ cf. Eq. (4),

$y=\left(\mu^{\times}-E_{\mathrm{D}}\right) / k T=\left(\mu^{\times}-E_{\mathrm{A}}\right) / k T$,

$e^{\eta}=b e^{y}$.

Two special cases shall be considered in some detail.

a) Vanishing hole density $p \approx 0, p_{2} \approx 0$.

Eq. (8) puts the Fermi level at

$$
\mu^{\times}=E_{\mathrm{D}}+k T \ln (\gamma / b)
$$

with *

$$
e^{\eta} \rightarrow \gamma=n_{\mathrm{D}} / n_{\mathrm{A}} \text {. }
$$

$\beta)$ Small hole density.

$e^{\eta}$ is slightly different from $\gamma$

$$
\begin{array}{r}
b e^{y}=e^{\eta}=\gamma(1+\varepsilon), \varepsilon \ll 1, \\
e^{-\eta}=1 / \gamma(1+\varepsilon) \approx(1-\varepsilon) / \gamma .
\end{array}
$$

Introducing * Eqs. (15) and (16) into (8), going to first order in $\varepsilon$ and assuming $p_{2} \ll n_{\mathrm{D}}$ yields

$\varepsilon=p_{2}(1+\gamma) /\left(\gamma n_{\mathrm{D}}+2 p_{2}\right) \approx p_{2}(1+\gamma) / n_{\mathrm{D}} \gamma$.

The result is for the hole density

$p=p_{2} e^{-\eta}=\left(n_{\mathrm{A}} / n_{\mathrm{D}}\right) p_{0} b \cdot \exp \left\{-\left(E_{\mathrm{S}}-E_{\mathrm{V}}\right) / k T\right\}$

$$
=n_{\mathrm{D}} \varepsilon /(1+\gamma) \text {, }
$$

for the density of ionized donors

$$
n_{\mathrm{D}}^{+}=\left[n_{\mathrm{A}} \gamma /(1+\gamma)\right][1-\varepsilon \gamma /(1+\gamma)],
$$

and for the density of ionized acceptors

$$
\begin{gathered}
n_{\mathrm{A}}^{-}=\left[n_{\mathrm{A}} \gamma /(1+\gamma)\right][1+\varepsilon /(1+\gamma)] . \\
p \ll n_{\mathrm{D}} \text { and } p \ll n_{\mathrm{A}}
\end{gathered}
$$

Note

because of the exponential factor in Equation (18). According to this same factor, with raising temperature, the hole density raises with the activation energy $\Delta k T=E_{\mathrm{S}}-E_{\mathrm{V}}$. On the contrary, the ionized donor density is nearly temperature independent.

As long as $k T \ll E_{\mathrm{S}}-E_{\mathrm{V}} \ll E_{\mathrm{L}}-E_{\mathrm{V}}$, there are no electrons in the conduction band under equilibrium conditions.

\footnotetext{
* In the more general case $a \neq 1 / b, \mu^{\times}=E_{\mathrm{D}}+k T y$ with $y$ from

$e^{y=}(1 / 2 b)\left\{\gamma-1+\left[\gamma^{2}+1+2 \gamma(2 a b-1)\right]^{1 / 2}\right\} . \quad$ (14a)

Then Eqs. (15) through (21) have to be changed accordingly.
} 


\section{c) Stationary State under Illumination at Low Temperature}

The transition rates $z$, cf. Fig. 2, the thermal transition probabilities $w$ and the densities in a stationary state are connected by

$$
\begin{aligned}
& z_{1}=w_{1} n_{\mathrm{A}}^{\times}=w_{2} n_{\mathrm{A}}^{-} p=z_{2}, \\
& z_{3}=w_{3} n_{\mathrm{D}}^{+}=w_{4} n_{\mathrm{D}}^{\times} p=z_{4}, \\
& z_{5}=w_{5} n_{\mathrm{A}}^{-} n_{\mathrm{D}}^{+}=w_{6} n_{\mathrm{D}}^{\times} n_{\mathrm{A}}^{\times}=z_{6} .
\end{aligned}
$$

Equilibrium values, marked in the following by a lower index g, can be taken from Eqs. (11) and (18) to $(20)$.

If now, by an external perturbation, the hole density is changed from $p_{\mathrm{g}}$ to $p$, this perturbation is transferred to $n_{\mathrm{A}}^{-}, n_{\mathrm{A}}^{\times}, n_{\mathrm{D}}^{+}$and $n_{\mathrm{D}}^{\times}$by the thermal processes only and a common quasi Fermi niveau is valid. With

$$
\frac{w_{4}}{w_{3}}=\frac{n_{\mathrm{Dg}}^{+}}{p_{\mathrm{g}} n_{\mathrm{Dg}}^{\times}}=\frac{n_{\mathrm{Dg}}^{+}}{p_{\mathrm{g}}\left(n_{\mathrm{D}}-n_{\mathrm{Dg}}^{+}\right)}=\frac{n_{\mathrm{D}}^{+}}{p\left(n_{\mathrm{D}}-n_{\mathrm{D}}^{+}\right)}
$$

and Eq. (7) $p$ is found as

$p=p_{\mathrm{g}} \frac{n_{\mathrm{D}}^{+}}{n_{\mathrm{Dg}}^{+}} \frac{n_{\mathrm{D}}-n_{\mathrm{Dg}}^{+}}{n_{\mathrm{D}}-n_{\mathrm{D}}^{+}}=p_{\mathrm{g}} \exp \left(\frac{\mu_{\mathrm{g}}^{\times}-\mu_{\mathrm{p}}^{\times}}{k T}\right)$.

There is an important special case with

(1) "a small number" of acceptors, viz. $n_{\mathrm{A}} \ll n_{\mathrm{D}}$ $\rightarrow \gamma \gg 1$;

(2) "medium" perturbation, viz. $n_{\mathrm{D}}^{+} \ll n_{\mathrm{D}}$.

Then

$$
p \approx p_{\mathrm{g}}\left(n_{\mathrm{D}}^{+} / n_{\mathrm{Dg}}^{+}\right) \approx p_{\mathrm{g}}\left(n_{\mathrm{D}}^{+} / n_{\mathrm{A}}\right) \text {, essentially } p \propto n_{\mathrm{D}}^{+} \text {. }
$$

If the perturbation of the hole density is brought about by absorption of light in the fundamental absorption region, there will be electrons in the conduction band

$$
n=p+n_{\mathrm{D}}^{+}-n_{\mathrm{A}}^{-} .
$$

For $p$ small, Eq. (22) with (19), (20) and (25) yield

$$
\begin{aligned}
w_{5} / w_{6} & \approx 1, \\
n_{\mathrm{A}}^{-} & =n_{\mathrm{A}}\left\{1-\left(n_{\mathrm{D}}^{+} / n_{\mathrm{D}}\right)\right\}, \\
n & =n_{\mathrm{D}}^{+}\left(1+p_{\mathrm{g}} / n_{\mathrm{Dg}}^{+}+n_{\mathrm{A}} / n_{\mathrm{D}}\right)-n_{\mathrm{A}} .
\end{aligned}
$$

Only for strong enough an excitation, $n_{\mathrm{D}}^{+} \gg n_{\mathrm{A}}$, the additive term $n_{\mathrm{A}}$ may be suppressed leaving

$$
n \propto n_{\mathrm{D}}^{+} .
$$

\section{d) Time Dependent Nonstationary Processes}

Now the scheme of Fig. 3 is used. The electrons on the levels $E_{\mathrm{A}}$ and $E_{\mathrm{D}}$ can be condensed into, viz. Eqs. (7), (19), (20) and (22 c)

$$
\begin{aligned}
S & =n_{\mathrm{D}}+n_{\mathrm{A}}, \\
S s & =n_{\mathrm{D}}^{+}+n_{\mathrm{A}}^{-} \quad \text { and }
\end{aligned}
$$

approximately ( $p$ small)

$$
n_{\mathrm{D}}^{+} / n_{\mathrm{A}}^{\times}=n_{\mathrm{D}} / n_{\mathrm{A}}=n_{\mathrm{D}}^{\times} / n_{\mathrm{A}}^{-} .
$$

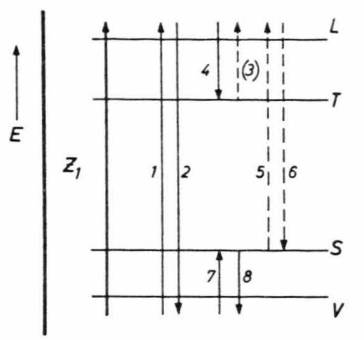

Fig. 3. Transitions in a nonequilibrium state. Symbols as in Figure 1. $Z_{1}$ optical induced transitions.

With $S s$ empty levels and $T \tau$ full traps the rate equations are

$\mathrm{d} p / \mathrm{d} t=Z_{1}+\ddot{u}_{1}-\ddot{u}_{2} n p+\ddot{u}_{7} S s-\ddot{u}_{8} S(1-s) p,(29)$

$\mathrm{d} n / \mathrm{d} t=Z_{1}+\ddot{u}_{1}-\ddot{u}_{2} n p+\ddot{u}_{3} T \tau-\ddot{u}_{4} T(1-\tau) n$,

$\mathrm{d} S s / \mathrm{d} t=-\ddot{u}_{7} S s+\ddot{u}_{8} S(1-s) p$,

$\mathrm{d} T \tau / \mathrm{d} t=-\ddot{u}_{3} T \tau+\ddot{u}_{4} T(1-\tau) n$.

$Z_{1}$ is the assumed optical generation rate. The coefficients $\ddot{u}$ are obviously not independent, but interrelated by the equilibrium densities. A numerical solution of Eqs. (29) through (32) will be presented in Section 4.

Here some analytical approximations will be discussed. Far from the saturation of $S$ and $T$ the relations

$$
\begin{aligned}
& s \ll 1, \quad 1-s \approx 1, \\
& \tau \ll 1, \quad 1-\tau \approx 1
\end{aligned}
$$

will be fulfilled.

As long as $T$ is nearly empty, the term $\ddot{u}_{3} T \tau$ may be suppressed. Using

$$
\overline{Z_{1}}=Z_{1}+\ddot{u}_{1},
$$

the rate equations are reduced to

$$
\begin{aligned}
& \mathrm{d} p / \mathrm{d} t=\bar{Z}_{1}-\ddot{u}_{2} n p+\ddot{u}_{7} S s-\ddot{u}_{8} S p, \\
& \mathrm{~d} n / \mathrm{d} t=\bar{Z}_{1}-\ddot{u}_{2} n p-\ddot{u}_{4} T n,
\end{aligned}
$$




$$
\begin{aligned}
& \mathrm{d} S s / \mathrm{d} t=-\ddot{u}_{7} S s+\ddot{u}_{8} S p, \\
& \mathrm{~d} T \tau / \mathrm{d} t=\ddot{u}_{4} T n .
\end{aligned}
$$

Elimination of $n$ and $p$ yields a differential equation for $s$

$$
\begin{aligned}
\left(\bar{Z}_{1}-S s\right) & \left(\dot{q}-\ddot{u}_{4} q T\right)+q S\left(\ddot{s}+\left(\ddot{u}_{2} / \ddot{u}_{8}\right) q \dot{s}\right) \\
& +\left(1 / \ddot{u}_{8}\right)\left(q \ddot{q}-\dot{q}^{2}\right) \\
& +\left(1 / \ddot{u}_{8}\right) q \dot{q}\left(\left(\ddot{u}_{2} / \ddot{u}_{8}\right) q+\ddot{u}_{4} T\right)=0
\end{aligned}
$$

with

$\dot{s}=\mathrm{d} s / \mathrm{d} t, \ddot{s}=\mathrm{d}^{2} s / \mathrm{d} t^{2}, \quad q=\dot{s}+\ddot{u}_{7} s, \dot{q}=\mathrm{d} q / \mathrm{d} t$,

$\ddot{q}=\mathrm{d}^{2} q / \mathrm{d} t^{2}$.

Two special cases shall be considered.

(a) "Medium times". The time variations of $s$, $n$ and $p$ shall be small: $\dot{s} \approx 0, \dot{p} \approx 0, \dot{n} \approx 0$. How will proceed the occupation of $T$ ?

Eliminating $n$ and $p$ from

$$
\begin{aligned}
0 & =\overline{Z_{1}}-\ddot{u}_{2} n p, \\
0 & =\overline{Z_{1}}-\ddot{u}_{2} n p, \\
0 & =-\ddot{u}_{7} S s+\ddot{u}_{8} S p, \\
\mathrm{~d} T \tau / \mathrm{d} t & =\ddot{u}_{4} T n,
\end{aligned}
$$

yields $\mathrm{d} T \tau / \mathrm{d} t=\left(\ddot{u}_{4} / \ddot{u}_{2}\right)\left(\ddot{u}_{8} / \ddot{u}_{7}\right)(T / s) \overline{Z_{1}}$.

Electroneutrality requires

$$
\dot{n}+T \dot{\tau}=\dot{p}+S \dot{s} \text { or } T \dot{\tau} \approx S \dot{s} .
$$

The resulting equation for $s$

leads to the result

$$
s \dot{s}=\frac{\ddot{u}_{4}}{\ddot{u}_{2}} \frac{\ddot{u}_{8}}{\ddot{u}_{7}} \frac{T}{S} \overline{Z_{1}}
$$

$$
\begin{aligned}
& s=\left(s_{1}^{2}+\alpha_{1} \overline{Z_{1}} t\right)^{1 / 2} ; \quad s(0)=s_{1}, \\
& \tau=\left(\tau_{1}^{2}+\beta_{1} \overline{Z_{1}} t\right)^{1 / 2}+\tau_{2}
\end{aligned}
$$

with

$$
\begin{aligned}
& \alpha_{1}=2 \ddot{u}_{4} \ddot{u}_{8} T / \ddot{u}_{2} \ddot{u}_{7} S \approx 2 \ddot{u}_{4} s_{\mathrm{g}} T / \ddot{u}_{2} p_{\mathrm{g}} S, \\
& \beta_{1}=2 \ddot{u}_{4} \ddot{u}_{8} S / \ddot{u}_{2} \ddot{u}_{7} T \approx 2 \ddot{u}_{4} s_{\mathrm{g}} S / \ddot{u}_{2} p_{\mathrm{g}} T
\end{aligned}
$$

and two constants $\tau_{1}$ and $\tau_{2}$.

The nonoccupation $s$ and the occupation $\tau$ obey a square root law.

(b) "Strong excitation". With $Z_{1}$ large, the num. ber of holes in the valence band is large and the transition rate 8 in Fig. 3 will be large compared to $7 . \ddot{u}_{7} \approx 0$ is adequate; Eq. (40) changes into

$$
S \dot{s}^{2}-\left(\ddot{u}_{4} \ddot{u}_{8} / \ddot{u}_{2}\right) T \bar{Z}_{1}+\left(\ddot{u}_{4} \ddot{u}_{8} / \ddot{u}_{2}\right) T S \dot{s}=0
$$

leading to the solution

$$
\begin{aligned}
& s=s_{\mathrm{g}}+(\eta / S) t, \\
& \tau=(\eta / T) t, \\
& n=\eta / \ddot{u}_{4} T, \\
& p=\eta / \ddot{u}_{8} S .
\end{aligned}
$$

$\eta$ has to satisfy the equation

$$
0=\bar{Z}_{1}-\eta-\left(\ddot{u}_{2} / \ddot{u}_{4} T \ddot{u}_{8} S\right) \eta^{2}
$$

with the border line cases

$\eta \approx \bar{Z}_{1}, \quad$ if $\bar{Z}_{1} \ll \ddot{u}_{4} \ddot{u}_{8} T S / \ddot{u}_{2}$, (56)

$\eta \approx\left(\bar{Z}_{1} \ddot{u}_{4} \ddot{u}_{8} T S / \ddot{u}_{2}\right)^{1 / 2}$, if $\overline{Z_{1}} \gg \ddot{u}_{4} \ddot{u}_{8} T S / \ddot{u}_{2}$. (57)

Equations (51) through (54) are describing a process by which electrons are finally transferred from $S$ to $T$, by the influence of the illumination. If $\ddot{u}_{3}$ is small enough, the reverse process $T \rightarrow S$ is nearly missing.

\section{Comparison of Experiments with Predictions of the Model}

\section{a) The Model}

The single positively charged (ionized) donors are assumed to be responsible for the electron paramagnetic resonance, cf. Section 2 a. The conductivity may result from two processes.

Firstly, there is conduction by electrons in the conduction band and by holes in the valence band. A separation of these contributions will not be tried. Yet, as boron is always p-type, $\mu_{\mathrm{p}}>\mu_{\mathrm{n}}$ is assumed and the hole contribution is taken as the dominant one.

Secondly, hopping conduction running over the donors D is assumed. Because of the presence of acceptors A, the donors are only partly occupied, cf. Section 2 a. Besides, the occupation of $\mathrm{D}$ depends on the hole concentration $p$, cf. Eqs. (18) through (20).

The light shall create electron hole pairs directly, $Z_{1}$ per unit of time and volume, cf. Figure 3.

\section{b) The Experimental Results ${ }^{1,7}$, *}

Only the relevant parts of the final results of the previous work $1, *$ are quoted.

Immediately with the onset of the illumination the electron and hole concentration $n$ and $p$ will

\footnotetext{
* See appendix.
} 
raise; the photoconductivity jumps to a starting value.

Afterwards, the enlarged hole concentration draws electrons from the $S$ levels, thus raising $n_{\mathrm{A}}^{\times}$and $n_{\mathrm{D}}^{+}$ $\left(n_{\mathrm{D}}^{+} \gg n_{\mathrm{A}}^{\times}\right)$and therefore also raising the spin concentration $n_{\mathrm{D}}^{+}$and the hopping conduction via the donors D. The experiments show a "fast photo-EPR" and a "fast photoconduction" with a time constant (see Appendix, c)

$$
1 / \ddot{u}_{7} \approx 1 \mathrm{~s} .
$$

Finally the electrons in the conduction band fall into the traps $\mathrm{T}$; there they are captured at low temperatures; they cannot leave the traps (exactly: they can leave them extremely slowly). These electrons are missing in S resp. D and are enlarging the concentration of spins $n_{\mathrm{D}}^{+}$and also the hopping conductivity via the levels $\mathrm{D}$. The experiments show a "persistent photo-EPR" and a "persistent photonconduction".

At last, the hopping conduction is assumed to be dominant at low temperatures, but the band conduction at high ones.

\section{c) Time and Illumination Dependence of the Photo-EPR at $77 \mathrm{~K}$}

According to the computations it is assumed, that a stationary state will not be reached in the time available. The traps $\mathrm{T}$ will be filled up slowly and the occupation of $\mathrm{S}$ changes according to Eq. (46)

$$
s=\left(s_{1}^{2}+\alpha_{1} \bar{Z}_{1} t\right)^{1 / 2} \approx\left(s_{1}^{2}+\alpha_{1} Z_{1} t\right)^{1 / 2} .
$$

Among the $s S$ unoccupied places there are, cf. Eq. (28)

$$
n_{\mathrm{D}}^{+} \approx s n_{\mathrm{D}}
$$

ionized, EPR effective donors.

Figures 4 and 5 demonstrate, that Eq. (58) succeeds pretty nice describing the experiments with two common constants. Some rather small residual differences may result from the approximations in the calculations as well as from the uncertainties in converting the measured spin numbers in concentration because of the inhomogeneous distribution of the optical excitation.

Figure 6 is an example of the linear dependence of $s$ on $t$, cf. Eq. (51)

$s_{\mathrm{g}}$ is negligible.

$$
s \propto n_{\mathrm{D}}^{+} \propto t
$$

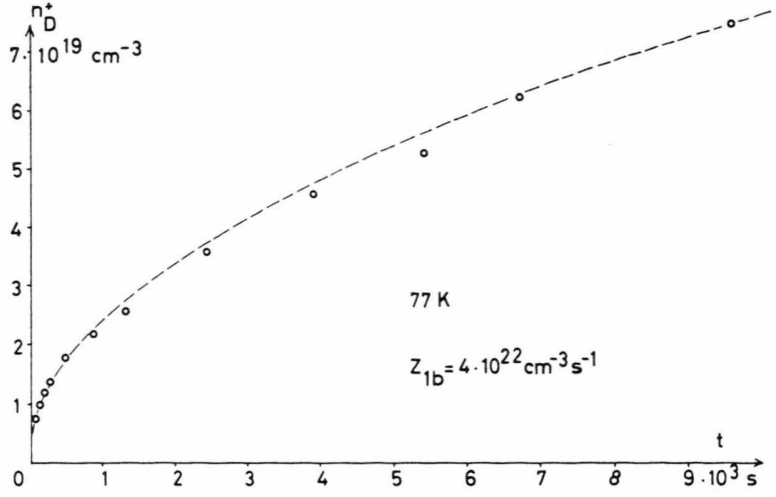

Fig. 4. Time dependence of the photo-EPR under illumination at $77 \mathrm{~K}$. Exciting wavelength between 0.63 and $1 \mu \mathrm{m}$; tungsten lamp $114 \mathrm{w} . Z_{1 \mathrm{~b}}=4 \times 10^{22} \mathrm{~cm}^{-3} \mathrm{~s}^{-1}$ quanta $\mathrm{ab}$ sorbed beneath the surface. $\bigcirc$ experimental values for the spin concentration $n_{\mathrm{D}}^{+}$beneath the surface. - - - values calculated according to Equation (58). $a_{1 \mathrm{~g}}=0.26 \times 10^{-29} \mathrm{~cm}^{3}, \mathrm{~s}_{1 \mathrm{~g}}^{2}=1.21 \times 10^{-6}$.

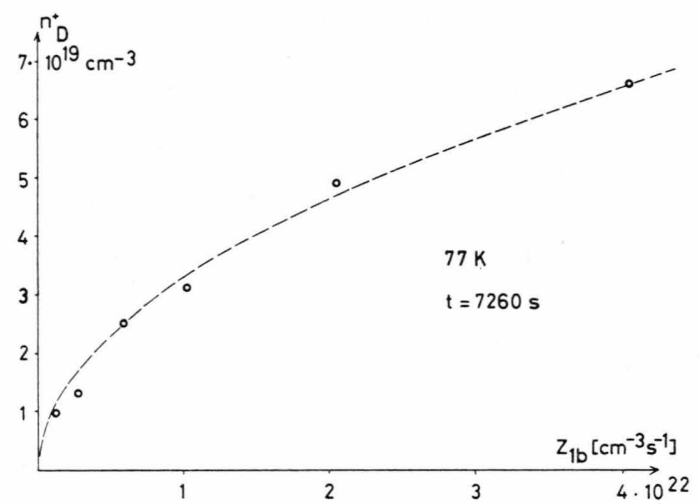

Fig. 5. Illumination dependence of the photo-EPR at $77 \mathrm{~K}$ after $t=7260 \mathrm{~s}$. Wavelengths see Figure 4. $\bigcirc$ experimental values for the spin concentration $n_{\mathrm{D}}^{+}$beneath the surface. - - values calculated according to Equation (58). The same constants as for Figure 4.

\section{d) Temperature Dependence of the Photo-EPR and Photoconductivity}

As discussed above, at $77 \mathrm{~K}$ there is persistent EPR. On the other hand, at $300 \mathrm{~K}$ after about $10 \mathrm{~s}$ a stationary state is reached. The temperature separating the two types of behaviour is about $T_{\mathrm{u}}=$ $150 \mathrm{~K}$. At $T_{\mathrm{u}}, n_{\mathrm{D}}^{+}$may change with a time constant of about $1 \mathrm{~h}$.

Further on, $T_{\mathrm{u}}$ is the temperature at which the two contributions to the photoconductivity, cf. Section $3 \mathrm{a}$, have approximately the same values. If there are under illumination $n=v p$ electrons and $p$ holes in the bands, both proportional to $n_{\mathrm{D}}^{+}$, Eqs. (25) and $(27 \mathrm{~b})$, then the "band contribution" is

$$
\sigma_{\mathrm{bd}}=e_{0} \mu_{\mathrm{p}} p\left(1+\mu_{\mathrm{n}} v / \mu_{\mathrm{p}}\right) \text {. }
$$




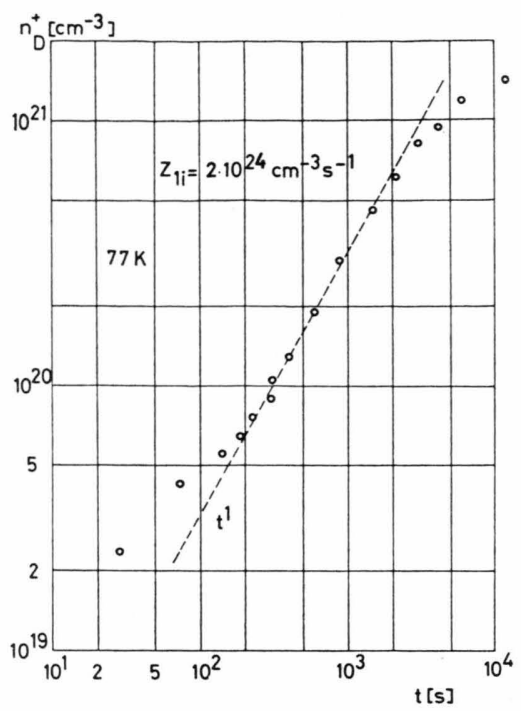

Fig. 6. Linear time dependence of the photo-EPR with strong excitation. Exciting wavelengths ${ }^{7}$ between UV and $2.7 \mu \mathrm{m}$. $77 \mathrm{~K} . Z_{1 \mathrm{w}}=2 \times 10^{24} \mathrm{~cm}^{-3} \mathrm{~s}^{-1}$. $\mathrm{n}_{\mathrm{D}}^{+}$spin concentration beneath the surface. In the linear range $\eta=\mathrm{d} n n^{+} / \mathrm{d} t=$ $2.75 \times 10^{17} \mathrm{~cm}^{-3} \mathrm{~s}^{-1}$.

The hopping contribution on the other hand will be

$$
\sigma_{\mathrm{hp}}=e_{0} \mu_{\mathrm{D}} n_{\mathrm{D}}^{+} \text {. }
$$

(1) Below $T_{\mathrm{u}}$, only $\sigma_{\mathrm{hp}}$ is important ( $p$ is too small). $\mu_{\mathrm{D}}$ is dependent both on the temperature ${ }^{1}$ and ${ }^{*}$ the ionized donor density $n_{\mathrm{D}}^{+}$

$$
\mu_{\mathrm{D}}\left(T, n_{\mathrm{D}}^{+}\right) \approx \mu_{0}(T)\left(n_{\mathrm{D}}^{+}\right)^{5 / 2} \text {. }
$$

Figure $7, T=150 \mathrm{~K}, n_{\mathrm{D}}^{+}=1.25 \times 10^{19} \mathrm{~cm}^{-3}$ and $\sigma_{\mathrm{u}}=5 \times 10^{-7}(\Omega \mathrm{cm})^{-1}$ yield

$$
\mu_{\mathrm{D}}=2.5 \times 10^{-7} \mathrm{~cm}^{2} / \mathrm{Vs} .
$$

At constant spin number, Fig. $7, \mu_{\mathrm{D}}$ obeys the law

$$
\mu_{\mathrm{D}}(T) \propto \exp \left\{-\Delta E_{\mu} / k T\right\}
$$

with $\Delta E_{\mu}=0.06 \ldots 0.09 \mathrm{eV}$. This dependence indicates a hopping mechanism.

(2) Above $T_{\mathrm{u}}$, band conduction is dominant. With $n_{\mathrm{A}} \ll n_{\mathrm{D}}$ and $n_{\mathrm{D}}^{+} \ll n_{\mathrm{D}}$ in any case

$$
\begin{aligned}
p=\left(n_{\mathrm{D}}^{+} / n_{\mathrm{D}}\right) p_{0} b & \cdot \exp \left\{-\left(E_{\mathrm{S}}-E_{\mathrm{V}}\right) / k T\right\} \\
& =s p_{0} b \cdot \exp \{-\Delta / k T\},
\end{aligned}
$$

[without illumination Eq. (18), with illumination Eqs. (24) or (25) with (18) ]. As $\mu_{\mathrm{p}}$ is only weakly temperature dependent, $\sigma_{\mathrm{bd}}(T)$ in Fig. 7 means

$$
\Delta=E_{\mathrm{S}}-E_{\mathrm{V}} \approx 0.21 \mathrm{eV} \text {. }
$$

* In Ref. ${ }^{1}$ a less precise estimate $\mu_{\mathrm{D}} \propto\left(n_{\mathrm{D}}{ }^{+}\right)^{4 / 2}$ had been given. - In Ref. ${ }^{3} n_{\mathrm{D}}{ }^{+}$is called $n$.

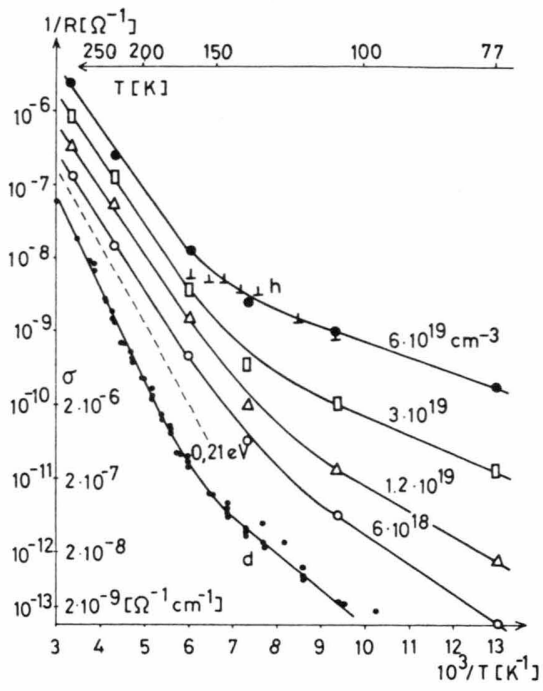

Fig. 7. Temperature dependence of the photoconductivity $\sigma$. Dark conductance $\mathrm{G}=1 / R$ (curve $\mathrm{d}$ ) and photoconductance $G=1 / R$ at constant spin concentration $n_{D^{+}}$(indicated at the curves). Transformation of the values by $n_{\mathrm{D}}{ }^{+}(x=0)=u N_{\mathrm{D}}{ }^{+}$ and $\sigma_{\mathrm{D}}(x=0)=r G$ (in the temperature range of hopping conduction). The transformation takes care of the inhomogeneous spin distribution in the sample ${ }^{1}$. $L$ length, $b$ width of the sample. $\mathrm{K}=2.5 . f(x)$ has been deduced from the

\begin{tabular}{|c|c|c|c|}
\hline$T(\mathrm{~K})$ & 300 & 150 & 77 \\
\hline$\frac{E_{\mathrm{L}}-E_{\mathrm{V}}}{2 k T}=\frac{\Delta E}{2 k T}$ & 29.6 & 60.1 & 117.4 \\
\hline $\begin{array}{c}\frac{E_{\mathrm{L}}-E_{\mathrm{T}}}{k T}=\frac{\Delta \mathrm{Tr}}{k T} \\
\left(\Delta_{\mathrm{Tr}}=0.36 \mathrm{eV}\right)\end{array}$ & 13.9 & 27.8 & 54.2 \\
\hline $\begin{array}{l}\frac{E_{\mathrm{D}}-E_{\mathrm{V}}}{k T}=\frac{\Delta}{k T} \\
(\Delta=0.23 \mathrm{eV})\end{array}$ & 8.88 & 17.8 & 34.6 \\
\hline $\exp (-\Delta E / 2 k T)$ & $1.38 \times 10^{-13}$ & $7.94 \times 10^{-27}$ & $10^{-51}$ \\
\hline $\exp \left(-\Delta_{\mathrm{Tr}} / k T\right)$ & $9.12 \times 10^{-7}$ & $8.32 \times 10^{-13}$ & $2.95 \times 10^{-24}$ \\
\hline $\exp (-\Delta / k \mathrm{~T})$ & $1.40 \times 10^{-4}$ & $1.90 \times 10^{-8}$ & $9.33 \times 10^{-16}$ \\
\hline$n_{03}=p_{03}\left(\mathrm{~cm}^{-3}\right)$ & $1.30 \times 10^{20}$ & $4.59 \times 10^{19}$ & $1.69 \times 10^{19}$ \\
\hline$n_{i 3}=p_{i 3}\left(\mathrm{~cm}^{-3}\right)$ & $1.79 \times 10^{7}$ & $3.64 \times 10^{-7}$ & $1.69 \times 10^{-32}$ \\
\hline$\mu_{\mathrm{p}}\left(\mathrm{cm}^{2} / \mathrm{Vs}\right)$ & 240 & 680 & 1840 \\
\hline $\begin{array}{l}E_{\mathrm{L}}-E_{\mathrm{V}}(\mathrm{eV}) \\
\text { Ref. } 10\end{array}$ & 1.533 & 1.555 & 1.560 \\
\hline \multicolumn{4}{|c|}{$\begin{array}{l}n_{03}=p_{03}=\left(2 / h^{3}\right)\left(2 \pi m^{\times} k T\right)^{3 / 2} \\
m^{\times}=m_{\mathrm{n}}=m_{\mathrm{p}}=3 m ; m \text { free electron mass } \\
n_{\mathrm{i} 3}=p_{\mathrm{i} 3}=n_{03} \exp (-\Delta E / 2 k T) \\
\text { temperature dependence }{ }^{10} \text { of } \Delta E: \Delta E(0)-3.24 \times 10^{-7} T^{2} ; \\
\quad \Delta E(0)=1.562 \mathrm{eV}\end{array}$} \\
\hline
\end{tabular}
optical absorption ${ }^{1}$.

$n_{\mathrm{D}}{ }^{+}(x)=n_{\mathrm{D}}{ }^{+}(0) f(x), \quad \int_{\text {sample }} n_{\mathrm{D}^{+}}(0) f(x) \mathrm{d} x=N_{\mathrm{D}}{ }^{+}$, $r=(\mathrm{L} / b)\left[\int f^{K}+1(x) \mathrm{d} x\right]^{-1}, \quad \int f(x) \mathrm{d} x=7.57 \times 10^{-4} \mathrm{~cm}=u$, $\int f^{K+1}(x) \mathrm{d} x=7.42 \times 10^{-5} \mathrm{~cm}^{3}, b=0.27 \mathrm{~cm}, L=0.57 \mathrm{~cm}$. Curve h see text in the appendix (e).

Table 1. Equilibrium values. 
(3) The assumption

$$
\sigma_{\mathrm{hp}} \approx \sigma_{\mathrm{bd}} \quad \text { at } \quad T=T_{\mathrm{u}}
$$

allows an estimate of $p$ and $\mu_{\mathrm{p}}$ [Eqs. (64) and (61 a) ] :

$$
\begin{aligned}
& p \approx 2.3 \times 10^{9} \mathrm{~cm}^{-3} \quad(T=150 \mathrm{~K}), \\
& \mu_{\mathrm{p}} \approx 680 \mathrm{~cm}^{2} / \mathrm{Vs} \quad(T=150 \mathrm{~K}),
\end{aligned}
$$

if $\mu \propto T^{-\alpha}$, with $\alpha=3 / 2$ (lattice scattering), than

$$
\mu_{\mathrm{p}}=240 \mathrm{~cm}^{2} / \mathrm{Vs} \quad(T=300 \mathrm{~K}) .
$$

There has been assumed

$n_{\mathrm{D}} \approx S \approx 2.4 \times 10^{21} \mathrm{~cm}^{-3} \approx$ number of unit cells $/ \mathrm{cm}^{3}$, cf. Section 5 ;

$n_{\mathrm{A}} \approx 10^{-2} n_{\mathrm{D}} ; \quad S=n_{\mathrm{A}}+n_{\mathrm{D}} ;$

$m_{\mathrm{p}} \approx 3 m, \quad m$ free electron mass;

$p_{03} \approx 4.6 \times 10^{19} \mathrm{~cm}^{-3}$, Eq. (9);

$b=1 / 2, \quad$ of. Section $2 \mathrm{a}$ and $\mathrm{b}$;

$E_{\mathrm{D}}-E_{\mathrm{V}}=E_{\mathrm{S}}-E_{\mathrm{V}} \approx 0,23 \mathrm{eV}, \quad$ Eq. (65) and Table 1; $n_{\mathrm{D}}^{+}=1.25 \times 10^{19} \mathrm{~cm}^{-3}$, cf. Fig. 7 and Table 1 ; $\sigma_{\mathrm{u}}=5 \times 10^{-7}(\Omega \mathrm{cm})^{-1}, \quad$ cf. Fig. 7 ;

$\mu_{\mathrm{p}}=\mu_{\mathrm{n}} \quad$ and $\quad v=1$.

\section{Computer Solution of the Differential Equations of the Model}

Starting with the Eqs. (29) to (32), the values in Table 2 and in the legends to Figs. 8 and 9 a computer solution ${ }^{8}$ has been tried. In principle, an Eulerian procedure is adequate, but modifications are necessary because of the kind of the direction field. Parts of the results shown in Figs. 8 and 9 are giving the general behaviour, especially the ranges according to Eqs. (46) resp. (51) with (54). In view of the large number of parameters, a full

Table 2. Values used for the model; cf. Figs. 4, 8 and 9 and Section 4. Temperature $77 \mathrm{~K}$.

\begin{tabular}{ll}
\hline$S=2.4 \times 10^{21} \mathrm{~cm}^{-3}$ & $\ddot{u}_{2}=10^{5} \mathrm{~cm}^{3} \mathrm{~s}^{-1}$ \\
$n_{\mathrm{D}}=2.4 \times 10^{21} \mathrm{~cm}^{-3}$ & $\ddot{u}_{3}=3 \times 10^{-7} \mathrm{~s}^{-1}$ \\
$T=2.4 \times 10^{20} \mathrm{~cm}^{-3}$ & $\ddot{u}_{4} T=9.6 \times 10^{-2} \mathrm{~s}^{-1}$ \\
$p_{\mathrm{b}}=n_{\mathrm{b}}=29 \mathrm{~cm}^{-3}$ & $\ddot{u}_{7}=1 \mathrm{~s}^{-1}$ \\
& $\ddot{u}_{8}=3 \times 10^{-3} \mathrm{~cm}^{+3} \mathrm{~s}^{-1}$
\end{tabular}

fit has not been tried. Rather, it shall be shown, that the parameters choosen are compatible with a number of known quantities.

a) From the equilibrium condition $d / \mathrm{d} t=0$ with Eqs. (38) and (64) it is deduced

$\ddot{u}_{7} / \ddot{u}_{8}=p / s=p_{03} b \cdot \exp \{-\Delta / k T\}=7.9 \times 10^{3} \mathrm{~cm}^{-3}$

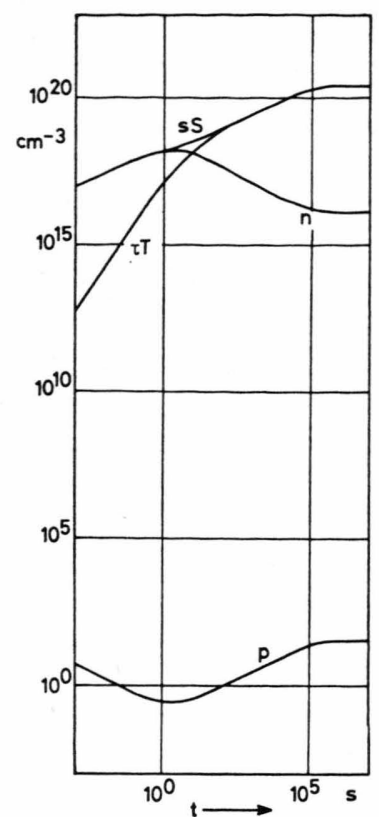

Fig. 8. Time dependences of the spin concentration $n_{\mathrm{D}}{ }^{+} \approx$ $s S$, the filled trap concentration $\tau T$, the hole concentration $p$, and the electron concentration $n$ according to Eqs. (29) to (32) as calculated by numerical solution ${ }^{8} \cdot Z_{1}=4 \times 10^{22} \mathrm{~cm}^{-3}$ $\mathrm{s}^{-1}$, all other values from Table 2 .

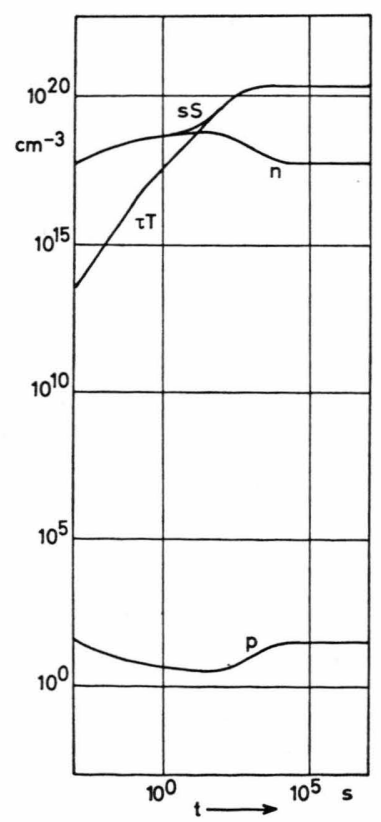

Fig. 9. $Z_{1}=2 \times 10^{24} \mathrm{~cm}^{-3} \mathrm{~s}^{-1}$, otherwise as Figure 8 .

(at $77 \mathrm{~K}$ from Table 1 ). In Table $2, \ddot{u}_{7} / \ddot{u}_{8}=\frac{1}{3} \times 10^{3}$ has been choosen. 
b) The time constant for a decay of $s S$, governed by $\ddot{u}_{7}$, Eq. (31) is lying near $1 \mathrm{~s}$ (Section $3 \mathrm{~b}$ and Appendix, c). Table 2 contains $\ddot{u}_{7}=1 \mathrm{~s}^{-1}$.

c) The value $\eta=\left(Z_{1} \ddot{u}_{4} T \ddot{u}_{8} S / \ddot{u}_{2}\right)^{1 / 2}$ can be taken from Fig. 6, giving $2.75 \times 10^{17} \mathrm{~cm}^{-3} \mathrm{~s}^{-1}$. The values from Table 2 lead to $3.7 \times 10^{18} \mathrm{~cm}^{-3} \mathrm{~s}^{-1}$.

d) $\mathrm{S}$ corresponds to the number of rhombohedral cells (Section 5).

e) $\alpha_{1}$ from Figs. 4 and 5 is $\alpha_{1}=2.6 \times 10^{-30} \mathrm{~cm}^{+3}$. Table 2 with Eq. (48) yields

$$
\alpha_{1}=2 \ddot{u}_{8} \ddot{u}_{4} T / \ddot{u}_{2} \ddot{u}_{7} S=2.4 \times 10^{-30} \mathrm{~cm}^{3} .
$$

The computer solution is suggesting simplifications in the equations. These have been used to derive analytical solutions for different time intervals, but they may be omitted here.

\section{Discussion}

The consistent description of the photo-EPR and photoconductivity in beta-rhombohedral single crystal boron by the model described represents one prerequisite for an understanding of boron. The concentration and the possible changes in the charge state of active centres - discussed in Section 2 a and used in $3 \mathrm{~d}$ and $4-$ are known. On the other hand final statements about their physical and chemical nature and on the kind of the charge transport, which they make possible, seem difficult.

In any case, it should be emphasized that the assumptions concerning the levels are compatible with the optical experiments (for a collection see ${ }^{10}$ ). In a new publication ${ }^{11}$, at the energy of $L$ in Fig. 1 localized states are assumed. Also if this assumption should finally prove correct, this would not make necessary essential changes in the model presented here.

As to the level S, the following considerations may be useful. The purity of the material, tested by chemical analysis, spectroscopical analysis and neutron activation analysis is so high, that foreign atoms should not be considered responsible for the centres $\mathrm{S}$ resp. D.

On the other hand, a certain concentration of $\mathrm{S}$ levels had to be assumed to explain the time and illumination dependence of the photo-EPR and photoconductance, of. Sections $3 \mathrm{~d}$ and 4 and Table 2. This concentration $\left(2.4 \times 10^{21} \mathrm{~cm}^{-3}\right)$ is just twice as large as the concentration of rhombohedral unit cells ${ }^{9}\left(1.216 \times 10^{21} \mathrm{~cm}^{-3}\right)$.
Therefore one might ask whether some peculiarity of the boron structure ${ }^{9}$ would be responsible for the $\mathrm{S}$ levels. The following observation may give a hint. The atoms of each icosahedron, may it lie at a corner or in the middle of an edge of the unit cell, undergo a certain number of bonds (one half bond by each of the two atoms making a bond) and this number is equal to the number of electrons. The situation is different for the central atom and the two "condensed systems" in every unit cell made up of parts of three icosahedra. These systems together with the central atom contain 171 electrons (from the outer shell) and 168 bonds. As the choice of the bonds allows for some ambiguity, the last figure might be enlarged (at most up to 192), but only in even steps (as two equivalent condensed systems are involved). Therefore, in no case the number of bonds and electrons would fit; this would be taken as an indication for a donor- or acceptor-like behaviour of the "condensed systems". With respect to the conduction mechanism it should be remembered, that these systems are linked together through the whole crystal, a fact, which might be important for carrier hopping motion.

\section{Summary}

Photoconductivity and photo-EPR (electron paramagnetic resonance) in beta-rhombohedral boron single crystals may be described, as far as the time-, illumination- and temperature-dependence is concerned, by the combined action of traps $(T=2.4$ $\times 10^{20} \mathrm{~cm}^{-3}$ ) with acceptor character below the conduction band $\left(E_{\mathrm{L}}-E_{\mathrm{T}}=0.36 \mathrm{eV}\right)$ and a level $\mathrm{S}$ above the valence band $\left(E_{\mathrm{S}}-E_{\mathrm{V}}=0.23 \mathrm{eV}\right)$ made up simultaneously of donors $\left(n_{\mathrm{D}}=2.4 \times 10^{21} \mathrm{~cm}^{-3}\right)$ and acceptors $\left(n_{\mathrm{A}}=2.4 \cdot 10^{19} \mathrm{~cm}^{-3}\right)$, cf. Table 1 . The ionized donors are responsible for the EPR.

Estimates for the transition probabilities deduced from a solution of the four coupled nonlinear differential equations are collected in Table 2. The hole mobility in the valence band may be about $600 \mathrm{~cm}^{2}$ per Vs at $150 \mathrm{~K}$. Hopping conduction via the $\mathrm{S}$ levels occurs with a density and temperature dependent mobility of about $2.5 \times 10^{-7} \mathrm{~cm}^{2} / V$ s (cf. Section $3 \mathrm{~d})$. The peculiar arrangement of bonds in the boron structure seems to be important for the nature of the $\mathrm{S}$ levels. 


\section{Acknowledgement}

Thanks are due to the Deutsche Forschungsgemeinschaft for efficient support of this work by measuring equipment.

\section{Appendix}

Photo Electron Paramagnetic Resonance (PhotoEPR) and Photoconductivity in Zone Molten Pure Beta-rhombohedral Boron Single Crystals. (Extract from the thesis ${ }^{7}$ of J. Meyer, not published. Work performed at the Institut für Angewandte Physik der Technischen Universität Clausthal.)

(a) The material used have been beta-rhombohedral boron single crystals, zone molten, with $\varrho=$ $2.6 \times 10^{6} \Omega \mathrm{cm}$ at $300 \mathrm{~K}$. Between $g=10$ and 0.85 only one EPR-line in the dark is present with $g=$ $2.0029 \pm 0.0001$ and a line width $\Delta B=1.8 \times 10^{-4} \mathrm{~T}$ ( $\triangle B$ full width between the extrema of the differentiated absorption line).

Alloyed gold contacts were used for the conductance measurements. The current-voltage dependence is linear at $300 \mathrm{~K}$ and follows

$$
i=A u^{x} \quad(4<u<150 \mathrm{~V})
$$

with $x=1.1 \ldots 1.3$ (higher values with stronger illumination) at $77 \mathrm{~K}$. This law is a property of the bulk material and not of the contacts.

(b) Method of measurement. The number of spins has been determined by a double integration of the digital measured differentiated EPR absorption line at $10 \mathrm{GHz}$; copper sulphate has been used as a standard. Illumination of the sample was performed with the filtered radiation of a tungsten lamp. "Red light" in Figs. A1, A2, A3, and A4 means radiation essentially in the range $1000 \ldots 630 \mathrm{~nm}$, "White light" radiation from about $2700 \mathrm{~nm}$ until the limit of transmission of the focussing glass lens at the beginning of the UV.

(c) Experimental results at $77 \mathrm{~K}$. Figure $\mathrm{Al}$ shows some EPR spectra in the dark and under illumination. The sharp line present in the dark is unaffected by the light. At the highest spin numbers (produced by long illumination with white light) the width of the photo-EPR-line decreases to about $1 / 10$ of its largest value of $35 \times 10^{-4} \mathrm{~T}$, see Figure A2.

Spin number $N$ and photoconductance $G$ are reproduced for a continuous illumination in Figure $3 \mathrm{~A}$. Both $N$ and $G$ are rising with rising illumination. After an interruption of the illumination, there is at first only a small fast decrease of $N$ and $G$. Most of the photo-EPR and photoconductance is a
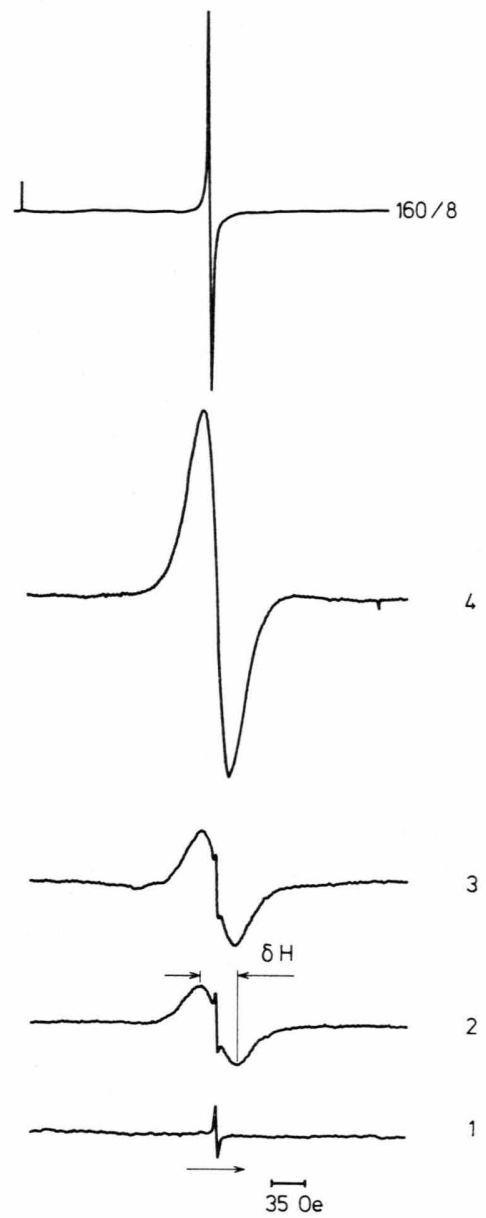

Fig. Al. Photo-EPR in beta-rhombohedral boron. Differentiated absorption line. $77 \mathrm{~K}$.

\begin{tabular}{|c|c|c|c|c|}
\hline $\begin{array}{l}\text { Curve } \\
\text { No. }\end{array}$ & Sample & $\begin{array}{l}\text { Amplifica- } \\
\text { tion }\end{array}$ & $\begin{array}{l}\text { Wavelength } \\
\text { range, } \\
\text { see (b) }\end{array}$ & $\begin{array}{l}\text { Duration of } \\
\text { the illumina- } \\
\text { tion }\end{array}$ \\
\hline \multirow{4}{*}{$\left.\begin{array}{l}1 \\
2 \\
3 \\
4 \\
160 / 8\end{array}\right\}$} & \multirow{3}{*}{$\begin{array}{l}\text { P72-15.2 } \\
\text { single } \\
\text { crystal }\end{array}$} & \multirow{3}{*}{$\times 200$} & \multirow[t]{3}{*}{ dark } & \multirow{4}{*}{$\begin{array}{l}-\overline{10} \min \\
25 \mathrm{~min} \\
250 \mathrm{~min} \\
18,5 \text { hours }\end{array}$} \\
\hline & & & & \\
\hline & & & & \\
\hline & $\begin{array}{l}12 \mathrm{Me} 1.1 \\
\text { polycrystal }\end{array}$ & $\times 8$ & "white" & \\
\hline
\end{tabular}

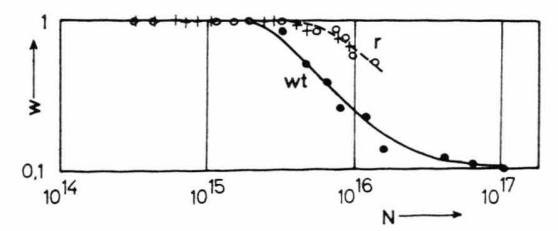

Fig. A2. Photo-EPR line width $w=\delta B / \delta B_{\max }$ as a function of the photo-spin-number $N . \delta B$ line width between the extrema of the differentiated absorption line. $\mathrm{r}$ red, wt white light, see (b). 


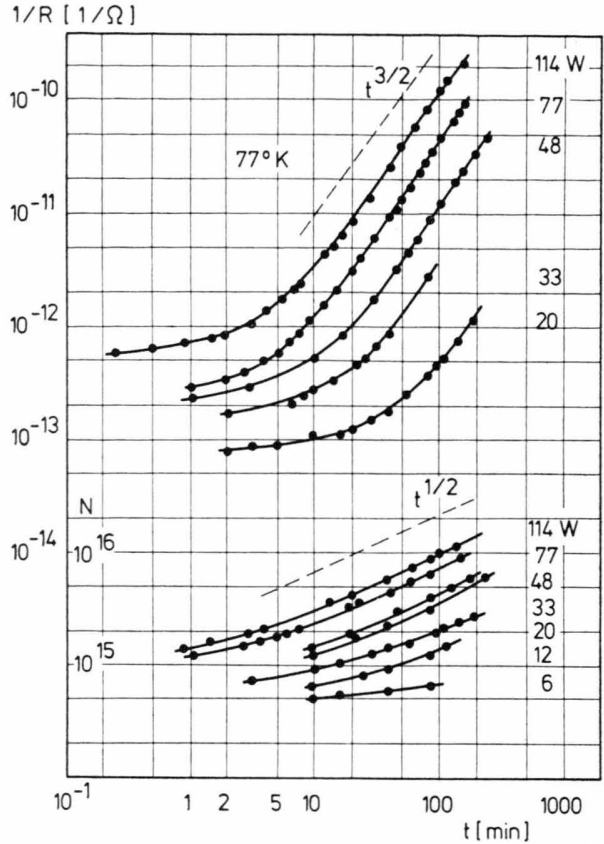

Fig. A3. Photo spin number $N_{\mathrm{p}}=N$ and photoconductance $G_{\mathrm{p}}=1 / R$ (persistent) of single crystal beta-rhombohedral boron versus the time of illumination $t .77 \mathrm{~K}$. Sample P 7215.2 and P 72-15.1. Parameters at the curves: power of the illuminating lamp. "Red light", see (b).

persistent effect, this means, $N$ and $G$ are decreasing with a time constant much larger than an hour (after the small initial drop).

The behaviour of the time dependent effects is shown in Figure A4. In any case, $t$ gives the total time of illumination.

Full points and full lines then belong to spin numbers $N$ and photoconductances $G$ measured under illumination; they give the "total effect".

Open points and broken lines result from measurements after an interruption of the illumination. These values, representing the "persistent effect", are reached with a time constant of about $8 \mathrm{~s}$.

If the illumination is turned on again, $N$ and $G$ rise to the values, which have been present just before the interruption of the illumination; with a time constant of about $1 \mathrm{~s}$. The difference between full and open points therefore represents the "fast effect".

The total effect and the persistent effect are independent of the sum of the length of any dark intervals, as long as this sum is short compared with the time constant of the decrease of the persistent effect (which is much larger than one hour). Therefore it was sufficient to use the time of illumination $t$ as abscissa in Figure A4.

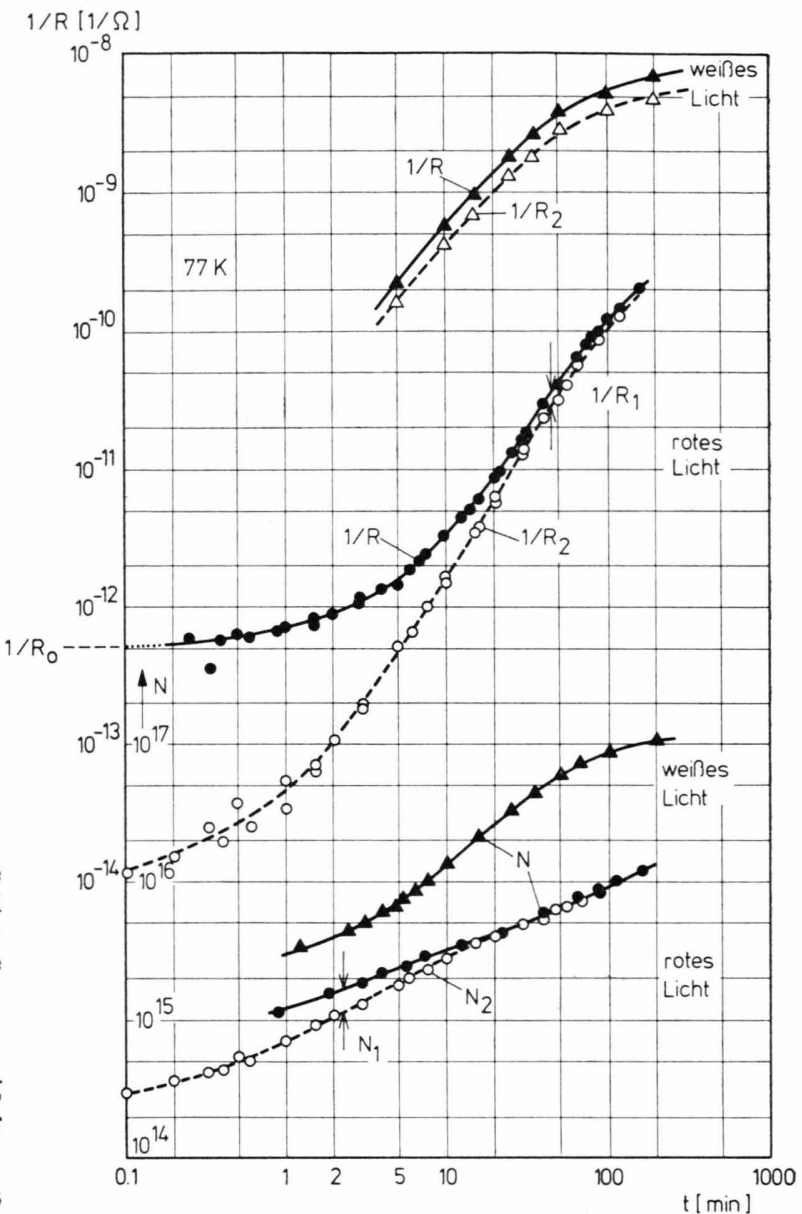

Fig. A4. Photo spin number $N$ and photoconductance $G=$ $1 / R$ of single crystal beta-rhombohedral boron versus the total duration of the illumination. $77 \mathrm{~K}$. Sample P 72-15.2. "Rotes Licht" = red, "weißes Licht" = white light. Full points and solid lines: measurements with illumination on. Open points and dashed lines: measurements after an interruption of the illumination $\left(N_{\mathrm{p}}, G_{\mathrm{p}}\right)$.

The measurements yield the spin number $N$; to convert this number to the spin concentration $n_{\mathrm{D}}^{+}$, the following consideration has been used. The light entering the sample will be absorbed thereby creating the spins. For a single wavelength the absorption follows $f=\exp \left\{-K_{1} x\right\} \cdot \exp \left\{-K_{2} x\right\}$. The absorption constant $K_{1}(\lambda)$ which can be measured at small photon fluxes and for short illumination times has been taken from the literature ${ }^{12,13}$. This absorption is active in the spin generation. The other constant $K_{2}\left(\lambda, n_{\mathrm{D}}^{+}\right)$is described in the literature as well ${ }^{14,15}$. In the neighbourhood of the absorption edge, the range which is important here, it is dependent only weakly on the wavelength but strongly on the time of illumination. On the whole $K_{2}=K_{20} n_{\mathrm{D}}^{+}(x)$ has been assumed. If diffusion of the spin is weak 
enough, an assumption, which is correct for low temperatures, the concentration $n_{\mathrm{D}}^{+}(x)$ of spins generated in the depth $x$ under the surface will be

$$
\begin{gathered}
n_{\mathrm{D}}^{+}(x)=n_{\mathrm{D}}^{+} \exp \left\{-K_{2} x\right\} \\
\int_{h f=1.4 \mathrm{eV}}^{2.0}(h f)^{2} \exp \{-(h f / k T)\} K_{1} \exp \left\{-K_{1} x\right\} h \mathrm{~d} f \\
\int_{h f=1.4 \mathrm{eV}}^{2.0}(h f)^{2} \exp \{-(h f / k T)\} K_{1} h \mathrm{~d} f
\end{gathered}
$$

giving the spin number

$$
N=b \underset{x=0}{L} \int_{\mathrm{D}}^{d} n_{\mathrm{D}}^{+}(x) \mathrm{d} x .
$$

The frequency dependence of the illumination intensity sufficiently corresponds to the approximation choosen for Plancks distribution.

By numerical calculation, the surface concentration $n_{\mathrm{D}}^{+}$of the spins could be deduced from the measured spin number $N$ ( $L$ sample length, $b$ width, $d$ thickness). The number of incident quanta also has been computed from the temperature and emission of the tungsten lamp, the absorption by filters and the geometry of the illuminating beam.

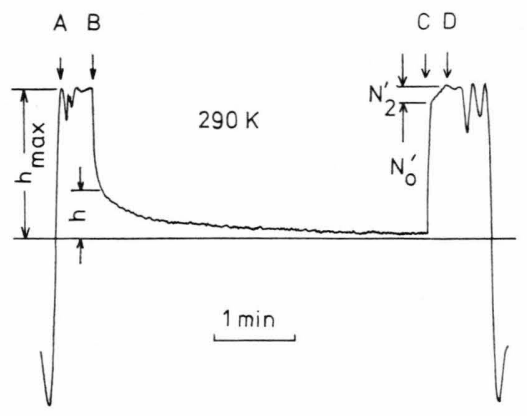

Fig. A5. Height of the photo-EPR-line as a function of time. $290 \mathrm{~K}$. Sample P 72-15.2. At B: light off. Time constants of the decrease $T_{1}<1 \mathrm{~s}, T_{2} \approx 120 \mathrm{~s}$. At C: light on. Time constants $T_{3}<1 \mathrm{~s}, T_{4} \approx 5 \mathrm{~s}$ (time constant of the recorder $\left.T_{\mathrm{r}}<1 \mathrm{~s}\right)$.

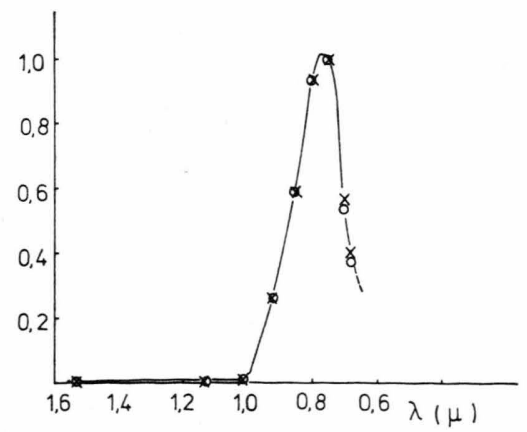

Fig. A6. Spectral dependences of the stationary values of the number of photospins $N_{\mathrm{st}}$ and of the photoconductance $G_{\mathrm{st}}=$ $1 / R_{\text {st }}\left(N_{\text {st }}\right.$ crosses, $G_{\text {st }}$ circles $)$ both normalized to the maximum. $300 \mathrm{~K}$. Boron single crystal, sample P 72-15.1. $\lambda$ wavelength in $\mu \mathrm{m}$.
The photoconductance has a "starting value" at the beginning of the illumination, and proportional to it. But it is negligibly small with respect to the fast and persistent effects just discussed.

(d) Experimental results at $293 \mathrm{~K}$. The time behaviour of the photo-EPR at this temperature is shown in Figure A5: within the accuracy of the measurements two time constants resp. (four altogether) are sufficient to describe the increase and the decrease of the number of photo-spins $N$, which is (in sections) approximately exponential. Then a stationary state is reached. The photoconductivity $G=1 / R$ reveals a similar behaviour.

The spectral dependences of $N_{\text {st }}$ and $G_{\text {st }}$ are the same, Fig. A6, and are consistent with ${ }^{2}$.

(e) Measurements at intermediate temperatures. As discussed under (c) and (d), at low temperatures the persisent effects are dominating whereas at high temperatures stationary values will be reached. At about $T_{\mathrm{u}}=150 \mathrm{~K}$, both effects are of comparable size. Therefore in Fig. A7, for $T>T_{\mathrm{u}}$ the quantities $N_{\text {st }}$ and $G_{\text {st }}$ on the one hand and for $T<T_{\mathrm{u}}, N_{\mathrm{p}}$ and $G_{\mathrm{p}}$ on the other are reproduced.

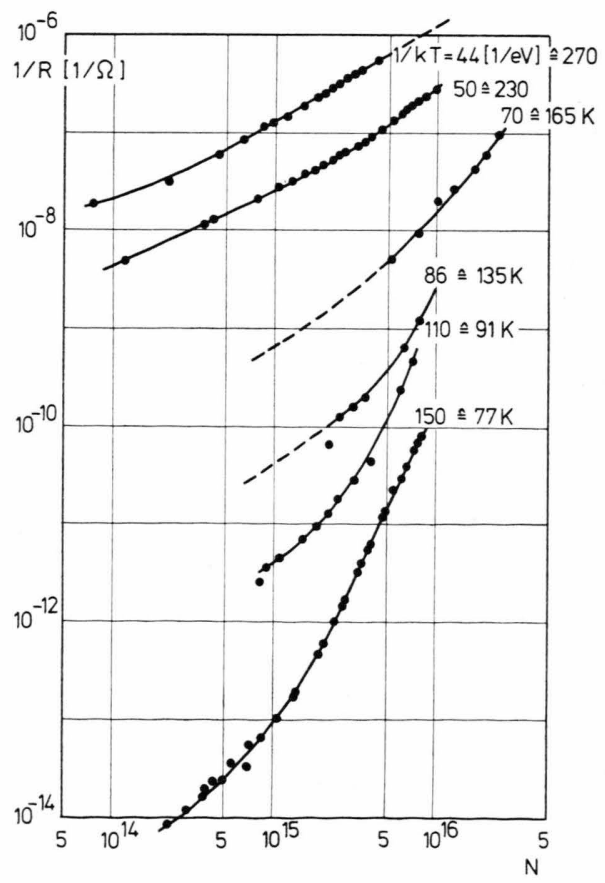

Fig. A7. Stationary spin number $N_{\text {st }}=N$ versus photoconductance $G_{\mathrm{st}}=1 / R$ for $T>T_{\mathrm{u}}=150 \mathrm{~K}$ and persistent spin number $N_{\mathrm{p}}=N$ versus photoconductance $G_{\mathrm{p}}=1 / R$ for $T<T_{\mathrm{u}}$. Dashed parts: extrapolated. Boron single crystal. 
From Fig. A7 the temperture dependence of the photoconductance at constant spin number has been derived and reproduced in Fig. 7 (of the main part). In the range above $T_{u}$ the activation energy of the conductance amounts to $\Delta E_{1} \approx 0.25 \mathrm{eV}$. In the range below $T_{\mathrm{u}} \Delta E_{2}$ is lying between 0.06 and $0.09 \mathrm{eV}$,

1 D. Geist and J. Meyer, Carrier Mobility in High Purity Boron Single Crystals. Proc. Xth Int. Conf. Physics Semiconductors, Cambridge, Mass. 1970, p. $597 \mathrm{ff}$.

2 D. Geist and W. Klein, XIV Colloque Ampère 1966. In Magnetic Resonance and Relaxation, 1967, p. 377. Ed. R. Blinc.

3 W. Klein and D. Geist, Z. Physik 201, 411 [1967].

4 D. Geist, J. Meyer, and H. Peussner, Electron Technology 3, 1/2, 207, Warszawa 1970.

5 W. Klein and D. Geist, Z. Physik 201, 411 [1967], Section $3 \mathrm{f}$ and 7 .

${ }^{6}$ D. Geist, Halbleiterphysik I, Vieweg, Braunschweig 1969, Chapter 13.2 (Boron and boron carbide).

7 J. Meyer, Photoleitung und Photoelektronenresonanz von beta-rhomboedrischen Bor-Einkristallen zwischen 77 und 300 K. Dissertation, Technische Universität Clausthal 1970.

8 Computations with H. Eckert, unpublished. depending on the spin number $N$. The low temperature parts of the curves may also be traced by generating a certain spin number at a low temperature by illumination (say $N=10^{16}$ ) and heating up slowly (curve h).

9 J. L. Hoard, R. E. Hughes, C. H. L. Kennard, D. E. Sands, D. B. Sullenger, and H. A. Weakliem, J. Amer. Chem. Soc. 85, 361 [1963]. - D. Geist, R. Kloß, and H. Follner, Acta Crystallographica B 26, 1800 [1970]. - Boron 3, 109 [1970]. Polish Scientific Publishers, Warsaw Conference 1968.

10 H. Werheit, Die Halbleitereigenschaften des Bors. In Solid State Problems X, 189 [1970]. Ed. O. Madelung, Vieweg, Braunschweig 1970.

11 A. J. Nadolny, IV Int. Conf. Boron, Tbilissi, Oct. 1972.

12 H. Werheit, Festkörperprobleme 10, 221 [1970].

13 Sh. Dzhamagidze and R. R. Shvangiradze, Fiz. Tek. Pol. 2. 382 [1968].

14 H. Werheit, Phys. Stat. Sol. 33, 587 [1969].

15 G. K. Gaulé, J. T. Breslin, and R. R. Pattey, Boron 2, 169 [1965], Plenum Press.

\title{
Ansiotropy of the Diffusion Coefficient in Nematic Liquid Solutions Measured by NMR Techniques
}

\author{
G. J. Krüger and H. Spiesecke \\ Magnetic Resonance Laboratory, Physics Division, EURATOM CCR, Ispra (Italy) \\ (Z. Naturforsch. 28 a, 964-967 [1973] ; received 16 February 1973)
}

\begin{abstract}
A gradient coil has been constructed for exact relative measurements of the longitudinal $\left(D_{\mathrm{L}}\right)$ and transverse $\left(D_{\mathrm{T}}\right)$ diffusion coefficients of nematic liquid crystals and of small molecules dissolved therein.

Some small molecules dissolved in the nematic phase of Licristal IV showed a $D_{\mathrm{L}} / D_{\mathrm{T}}$ ratio varying from about 1.1 to 1.25 , depending on the concentration. In contrast, tetramethylsilane dissolved in p-methoxy-benzylidene-p'-n-butyl-aniline showed no anisotropy in the nematic phase.
\end{abstract}

\section{Introduction}

Measurements of self diffusion coefficients in nematic liquid solutions have been reported in an earlier paper ${ }^{1}$. It was not possible, however, to substantiate the anisotropy of diffusion in a magnetic field. From the estimated accuracy of measurement it was concluded that the anisotropy could not be more than $30 \%$. A similar measurement has been published by Murphy and Doane ${ }^{2}$, who, however, revoked their results in a later communication ${ }^{3}$. To make an absolute measurement of two diffusion coefficients which differ by only $30 \%$ or even less is

Reprint requests to Dr. G. J. Krüger, Magnetic Resonance Laboratory, Physics Division, EURATOM-CCR, I-21020 Ispra (Varese)/Italy. rather difficult. A new measuring arrangement has therefore been constructed which allows rather accurate relative measurements in the longitudinal and transverse directions with respect to the magnetic field.

\section{Apparatus and Measurements}

The measurements were done by pulsed NMR techniques with pulsed field gradients at $48 \mathrm{MHz}$ using the $90^{\circ}-180^{\circ}$ spin echo ${ }^{4,5}$. The amplitude $M$ of the nuclear spin echo in the presence of large gradient pulses is given by ${ }^{5}$ :

$$
M=M_{0} \exp \left\{-\gamma^{2} D \delta^{2}\left(\Delta-\frac{1}{3} \delta\right) G^{2}\right\}
$$

where $M_{0}$ is the echo amplitude without gradient pulses, $\gamma$ gyromagnetic ratio of the nuclei, $D$ their 